\title{
Utilization of the cyclic interferometer in polarization phase-shifting technique to determine the thickness of transparent thin-films
}

\author{
RAPEEPAN KAEWON ${ }^{1}$, CHUTChai PAWONG ${ }^{2}$, RATCHAPAK CHITAREE ${ }^{3}$, \\ TOSSAPORN LERTVANITHPHOL ${ }^{4}$, APICHAI BHATRANAND ${ }^{5 *}$ \\ ${ }^{1}$ Silpakorn University, Faculty of Engineering and Industrial Technology, \\ Department of Electrical Engineering, 6 Rajamankha Nai Rd, Nakornpathom 73000, Thailand \\ ${ }^{2}$ Rajamangala University of Technology Krungthep, Faculty of Science and Technology, \\ Physics Division, 2 Nanlinji Road, Bangkok 10120, Thailand \\ ${ }^{3}$ Mahidol University, Faculty of Science, Department of Physics, \\ 272 Rama VI Road, Bangkok 10400, Thailand \\ ${ }^{4}$ National Electronics and Computer Technology Center, Optical Thin-Film Laboratory, \\ Pathumthani 12120, Thailand \\ ${ }^{5}$ King Mongkut's University of Technology Thonburi, Faculty of Engineering, \\ Department of Electronic and Telecommunication Engineering, \\ 126 Pracha U-thid Road, Bangkok 10140, Thailand \\ *Corresponding author: apichai.bha@mail.kmutt.ac.th
}

An alternative polarization phase-shifting technique is proposed to determine the thickness of transparent thin-films. In this study, the cyclic interferometric configuration is chosen to maintain the stability of the operation against external vibrations. The incident light is simply split by a non-polarizing beam splitter cube to generate test and reference beams, which are subsequently polarized by a polarizing beam splitter. Both linearly polarized beams are orthogonal and counter -propagating within the interferometer. A wave plate is inserted into the common paths to introduce an intrinsic phase difference between the orthogonal polarized beams. A transparent thin-film sample, placed in one of the beam tracks, modifies the output signal in terms of the phase retardation in comparison with the reference beam. The proposed phase-shifting technique uses a moving mirror with a set of "fixed" polarizing elements, namely, a quarter-wave retarder and a polarizer, to facilitate phase extraction without rotating any polarizing devices. The measured thicknesses are compared with the measurements of the same films acquired using standard equipment such as the field-emission scanning electron microscope and spectroscopic ellipsometer. Experimental results with the corresponding measured values are in good agreement with commercial measurements. The system can be reliably utilized for non-destructive thickness measurements of transparent thin -films.

Keywords: polarization phase-shifting technique, cyclic interferometric configuration, non-destructive thickness measurements, transparent thin-films. 


\section{Introduction}

Phase-shifting interferometry is generally composed of two major procedures - the phase shifting step and the phase extraction algorithm - in which the latter gives the phase information of a sample introduced in the interferometric system. The most common methods to generate phase shift are moving a mirror [1-4], displacing a diffraction grating [ $[\underline{5}]$, tilting a glass plate $[\underline{6}, \underline{7}]$, and rotating polarizing devices such as a phase plate [ $\underline{8}-\underline{12}]$, and a polarizer [ $\underline{13}-15]$. The choice of the phase-shifting method for a particular application depends on available optical components coupled with its advantages deemed appropriate for the implementation. In this study, a cyclic interferometric configuration, which is less prone to the instability due to environmental disturbances, is chosen $[\underline{16}, 17]$. The proposed phase-shifting technique used to generate phase shifts for the setup is based on the mirror displacement and the combination of a quarter-wave retarder and a polarizer. By integrating both units into the cyclic interferometer, an interesting feature, which cannot be obtained from either mirror displacement or the combination of a quarter-wave retarder and a polarizer, is achieved. Two orthogonal linearly polarized beams emitted from the cyclic interferometer are transformed by the combination of a quarter-wave retarder and a polarizer fixed at a pre-aligned orientation into two circularly polarized beams with opposite handedness. They then combine to give a linearly polarized beam whose electric vector has a particular orientation relative to a reference axis. It is well known that the modulated phase difference between the two circularly polarized beams leads to the rotation of the resultant polarized beam: the so-called rotating polarized light. The modulation can be achieved with the moving mirror attached to the piezoelectric transducer (PZT) whose speed can simply be controlled by the driving signal. The proposed technique results in the rotation of the signal outputs via modulation while the polarizing device, either a wave-phase retarder or a polarizer, is fixed. This actually is contrary to the normal procedure of the polarization phase-shifting method whereby either the wave-plate retarder or polarizer has to be rotated to achieve the phase shift.

To implement the proposed polarization phase-shifting technique, a homogeneous and transparent thin-film of $\mathrm{WO}_{3}$ is used as a test sample. The sample inserted in the optical path between the two mirrors, i.e. $\mathrm{M}_{1}$ and $\operatorname{PZT}\left(\mathrm{M}_{2}\right)$, directly introduces a phase shift in the reference beam. With the proposed extraction technique, which will be discussed later, the phase shift can simply be determined and subsequently converted into the corresponding thickness of the thin-film sample. To verify the obtained thickness, the sample thickness was also examined with thin-film measuring instruments such as a field-emission scanning electron microscope and a spectroscopic ellipsometer.

\section{Principle}

\subsection{Principle of a cyclic interferometer system without a sample}

The schematic of the cyclic interferometer (CI) system is illustrated in Fig. 1. The laser light source (LS) emits linearly polarized light, whose direction is parallel to the axis 


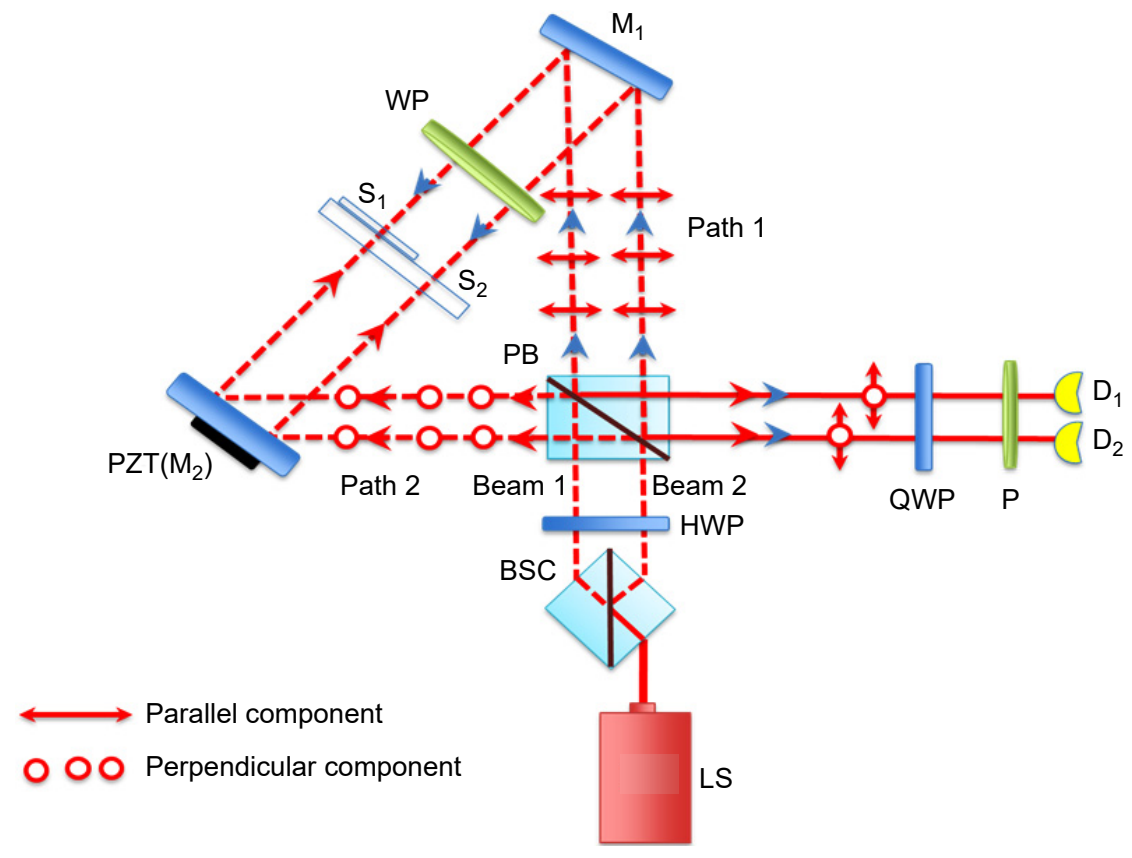

Fig. 1. Schematic of a cyclic interferometer.

of the polarization beam splitter (PB), reaching the CI. A beam splitter cube (BSC) placed after the laser is used to split an incident light beam into beam 1 and beam 2 . Both beams can be rotated to any commonly desired orientation by adjusting the halfwave plate (HWP). The HWP is oriented at $22.5^{\circ}$ with respect to the axis of the PB. This results in the polarization directions of both beams being at $45^{\circ}$ with respect to the axis of the PB. Therefore, the Jones vectors, representing the electric fields of beam 1 and beam 2 , are identical and given as

$$
E_{i}=\frac{1}{\sqrt{2}}\left[\begin{array}{l}
1 \\
1
\end{array}\right]
$$

At the PB, both polarized beams are equally split into two sets of orthogonal linearly polarized beams. Each polarized beam travels in perpendicular directions as guided by mirror $1\left(\mathrm{M}_{1}\right)$ and mirror $2\left(\mathrm{M}_{2}\right)$ that is attached to a PZT $\left(\operatorname{PZT}\left(\mathrm{M}_{2}\right)\right)$. The states of the twin polarized light beams with an electric field, as the parallel transmitted light beams travelling along path 1 , are identically linearly parallel to the plane of the interferometer. In contrast, the reflected light beams with an electric field propagating along path 2 , are identically linearly perpendicular to the plane of the interferometer. These dual polarized light beams from the two paths are then recombined at the $\mathrm{PB}$ and are transformed into two sets of circularly polarized light beams by the quarter-wave plate (QWP). The resulting twin beams are the so-called dual rotating polarized light (RPL). Both output beams, with electric fields $E_{i \mathrm{~T}}$ and $E_{i \mathrm{R}}$, in the two tracks can be equivalently expressed as 


$$
\begin{aligned}
& E_{i \mathrm{~T}}=\mathrm{QW} T_{\mathrm{PBT}} M_{\mathrm{R}} \mathrm{WP} M_{\mathrm{R}} T_{\mathrm{PBT}} E_{i}=\frac{1}{2}\left[\begin{array}{c}
\exp \left(i \delta_{\mathrm{F}}\right) \\
-i \exp \left(i \delta_{\mathrm{F}}\right)
\end{array}\right] \\
& E_{i \mathrm{R}}=\mathrm{QW} T_{\mathrm{PBR}} M_{\mathrm{R}} \mathrm{WP} M_{\mathrm{R}} T_{\mathrm{PBR}} E_{i}=\frac{1}{2}\left[\begin{array}{c}
-i \exp \left[i\left(\delta_{\mathrm{M}_{1}}+\delta_{\mathrm{M}_{2}}(t)+\delta_{\mathrm{S}}\right)\right] \\
\exp \left[i\left(\delta_{\mathrm{M}_{1}}+\delta_{\mathrm{M}_{2}}(t)+\delta_{\mathrm{S}}\right)\right]
\end{array}\right]
\end{aligned}
$$

Here, $T_{\mathrm{PBT}}$ and $T_{\mathrm{PBR}}$ denote the Jones matrices of the linear PB $[\underline{18}, \underline{19}]$. The phase shifts $\delta$ of the dual polarized light beams after reflections from mirror $\mathrm{M}_{1}$ and $\mathrm{PZT}\left(\mathrm{M}_{2}\right)$ are $\delta_{\mathrm{M}_{1}}$ and $\delta_{\mathrm{M}_{2}}(t)$, where $\delta_{\mathrm{M}_{2}}(t)$ is time-dependent. The Jones representation $M_{\mathrm{R}}$ of each mirror, a linear birefringent wave plate (WP), and the output quarter-wave plate (QWP) QW are given by following equations:

$$
\begin{aligned}
& M_{\mathrm{R}}=\left[\begin{array}{ll}
1 & 0 \\
0 & \exp (i \delta)
\end{array}\right] \\
& \mathrm{WP}=\left[\begin{array}{ll}
\exp \left(i \delta_{\mathrm{F}}\right) & 0 \\
0 & \exp \left(i \delta_{\mathrm{S}}\right)
\end{array}\right] \\
& \mathrm{QW}=\frac{1}{\sqrt{2}}\left[\begin{array}{rr}
1 & -i \\
-i & 1
\end{array}\right]
\end{aligned}
$$

where $\delta_{\mathrm{F}}$ and $\delta_{\mathrm{S}}$ are phase retardations represented by the fast and slow eigenmodes [20], respectively, and the QWP is oriented with its fast axis at $-45^{\circ}$ with respect to the transmission axis of the PB. Note that the vibrating PZT $\left(\mathrm{M}_{2}\right)$ is sinusoidally driven by a function generator, and the dual phase shifts introduced by $\mathrm{M}_{2}$ alternate with time. Generally, each pair of twin polarized light beams is equally vibrated by a PZT, and they are in phase. Hence, a general WP is placed between $\mathrm{M}_{1}$ and PZT $\left(\mathrm{M}_{2}\right)$ to produce an unbalanced phase shift through its fast and slow axes, so that the relative phases are changed within the CI system. Both the output beams have the same electric field $E_{\text {out }}$ that is equivalent to the summation of $E_{i \mathrm{~T}}$ and $E_{i \mathrm{R}}$ in the two tracks in the absence of a sample, and can be written as [19]

$$
\begin{aligned}
E_{\text {out }} & =\frac{1}{2}\left\{\left[\begin{array}{r}
1 \\
-i
\end{array}\right] \exp \left(i \delta_{\mathrm{F}}\right)+\left[\begin{array}{l}
1 \\
i
\end{array}\right] \exp \left[i\left(\delta_{\mathrm{S}}+\delta_{\mathrm{M}_{2}}(t)+\delta_{\mathrm{M}_{1}}-\frac{\pi}{2}\right)\right]\right\} \\
& =\left[\begin{array}{l}
\sin \left(\delta_{\mathrm{t}}(t)\right) \\
\cos \left(\delta_{\mathrm{t}}(t)\right)
\end{array}\right] \exp \left[\frac{i}{2}\left(\delta_{\mathrm{F}}+\delta_{\mathrm{S}}+\delta_{\mathrm{M}_{2}}(t)+\delta_{\mathrm{M}_{1}}-\frac{\pi}{2}\right)\right]
\end{aligned}
$$

with $\delta_{\mathrm{t}}(t)=\delta_{\mathrm{S}}+\delta_{\mathrm{M}_{1}}+\delta_{\mathrm{M}_{2}}(t)-\delta_{\mathrm{F}}$ representing the dual output phase shifts in the CI. Here, it is obvious that the mirror movement gives rise to the change in the phase shifts 
corresponding to the varying orientations of the dual RPL. Subsequently, the two output light beams after the QWP reach the polarizer $(\mathrm{P})$, and are separately detected by photodetector $1\left(\mathrm{D}_{1}\right)$ and photodetector $2\left(\mathrm{D}_{2}\right)$. The dual RPLs are automatically dual rotating with the fixed polarizer; the output intensities detected by $D_{1}$ and $D_{2}$ are expected to follow the variation described by Malus' law. Here, we note that the orientations of the parallel-polarized light beams are "rotating" with respect to the transmission axis of the polarizer. Hence, any phase shifts of interest will be introduced later by inserting $\mathrm{WO}_{3}$ thin-film samples on a BK7 glass substrate and purely BK7 glass substrate in the cavity composed of a sample track and reference track, respectively.

\subsection{Principle of $\mathrm{CI}$ with the presence of a sample}

The dual beams within the CI are employed as a set of the probing tools to investigate the phase shifts introduced by a sample placed between $\mathrm{M}_{1}$ and PZT $\left(\mathrm{M}_{2}\right)$, as depicted in Fig. 1. The substrate of the sample is BK7. The $\mathrm{WO}_{3}$ thin film is deposited on half of the BK7 substrate, and this sample part is called $\mathrm{S}_{1}$. The other half $\mathrm{S}_{2}$ is just a pure BK7 substrate. The sample is positioned in the CI system such that tracks associated with $S_{1}$ and $S_{2}$ are called sample and reference tracks, respectively. In the sample track, the electric fields of the polarized beams traveling along paths 1 and 2 are denoted by $E_{i \mathrm{~T}, \mathrm{~S}_{1}}$ and $E_{i \mathrm{R}, \mathrm{S}_{1}}$, respectively. The Jones matrix $S$ of the homogenous sample is expressed as $[\underline{18}, \underline{19}]$

$$
S=\left[\begin{array}{ll}
T_{1} & 0 \\
0 & T_{2} \exp \left(i \Delta_{\mathrm{S}_{i}}\right)
\end{array}\right]
$$

where $T_{1}$ and $T_{2}$ represent the transmission coefficients along the principal axes of the thin-film on the transparent sample and $\Delta_{\mathrm{S}_{i}}$ is the phase retardation introduced by the $\mathrm{S}_{i}$ track when $\mathrm{S}_{1}$ and $\mathrm{S}_{2}$ are inserted, respectively. Thus, the Jones vector $E_{i \mathrm{~T}, \mathrm{~S}}$ representing the transmitted light in the $S_{1}$ track can be expressed as

$$
E_{i \mathrm{~T}, \mathrm{~S}_{1}}=\frac{1}{2}\left[\begin{array}{c}
T_{1} \\
-i T_{1}
\end{array}\right] \exp \left(i \delta_{\mathrm{F}}\right)
$$

Likewise, $E_{i \mathrm{R}, \mathrm{S}_{1}}$, the Jones vector of the reflected light beam in the $\mathrm{S}_{1}$ track, is expressed as

$$
E_{i \mathrm{R}, \mathrm{S}_{1}}=\frac{1}{2}\left[\begin{array}{c}
-i T_{2} \\
T_{2}
\end{array}\right] \exp \left[i\left(\Delta_{\mathrm{S}_{1}}+\delta_{\mathrm{S}}+\delta_{\mathrm{M}_{2}}(t)+\delta_{\mathrm{M}_{1}}\right)\right]
$$

Therefore, the Jones vector $E_{\text {out, }}^{\theta}$, $_{1}$ of the $\mathrm{S}_{1}$ output beams, emerging from the CI setup, can be expressed as

$$
E_{\text {out }, \mathrm{S}_{1}}^{\theta}=P(\theta)\left[E_{i \mathrm{~T}, \mathrm{~S}_{1}}+E_{i \mathrm{R}, \mathrm{S}_{1}}\right]
$$


where $P(\theta)$ represents the Jones matrix of the linear polarizer $\mathrm{P}$ whose transmission axis is aligned at an angle $\theta$ with respect to the reference axis $[\underline{20}, 21]$. In this study, the polarizer is adjusted to align at an angle of $45^{\circ}$ to the reference axis. The RPL orientation at $0^{\circ}, 45^{\circ}$, and $90^{\circ}$ can be expressed as

$$
\begin{aligned}
& I_{\text {out, } \mathrm{S}_{1}}^{0^{\circ}}=\frac{T_{1}^{2}}{4}+\frac{T_{1} T_{2} \sin \left(\Delta_{\mathrm{S}_{1}}+\delta_{\mathrm{t}}(t)\right)}{2}+\frac{T_{2}^{2}}{4} \\
& I_{\text {out, } \mathrm{S}_{1}}^{45^{\circ}}=\frac{T_{1}^{2}}{4}+\frac{T_{1} T_{2} \cos \left(\Delta_{\mathrm{S}_{1}}+\delta_{\mathrm{t}}(t)\right)}{2}+\frac{T_{2}^{2}}{4} \\
& I_{\text {out, } \mathrm{S}_{1}}^{90^{\circ}}=\frac{T_{1}^{2}}{4}-\frac{T_{1} T_{2} \sin \left(\Delta_{\mathrm{S}_{1}}+\delta_{\mathrm{t}}(t)\right)}{2}+\frac{T_{2}^{2}}{4}
\end{aligned}
$$

Thus, the phase retardation $\Delta_{\mathrm{S}_{1}}$, introduced by an inserted $\mathrm{WO}_{3}$ thin-film sample in the $S_{1}$ track, can be obtained from Eqs. (12) to (14) as

$$
\Delta_{\mathrm{S}_{1}}+\delta_{\mathrm{t}}(t)=\tan ^{-1}\left(\frac{I_{\text {out, } \mathrm{S}_{1}}^{0^{\circ}}-I_{\text {out, }}^{90_{1}^{\circ}}}{2 I_{\text {out, } \mathrm{S}_{1}}^{45^{\circ}}-I_{\text {out, } \mathrm{S}_{1}}^{90^{\circ}}-I_{\text {out, } \mathrm{S}_{1}}^{0^{\circ}}}\right)
$$

The RPL orientation depends on the mirror vibration. Subsequently, the output light after QWP goes into the polarizer $\mathrm{P}$ and the photodetector $\mathrm{D}_{1}$. The rotation of the linearly polarized output beam with respect to a static linear polarizer automatically allows the orientation of the beam caused by the initial phase from the apparatus to be offset. This action is marked by a time called the offset time, which indicates a new starting time for a subsequently introduced phase shift. Therefore, the both beams of phase shifts will be introduced later by a thin-film sample which is measured relative to the offset times. The initial phase shift $\delta_{t}(t)$ can then be offset. The polarization state after QWP is rotated from its original orientation as a result of the additional retardation caused by the sample. Thus, Eq. (15) can be rewritten as

$$
\Delta_{\mathrm{S}_{1}}=\tan ^{-1}\left(\frac{I_{\text {out, } \mathrm{S}_{1}}^{0^{\circ}}-I_{\text {out, } \mathrm{S}_{1}}^{90^{\circ}}}{2 I_{\text {out, } \mathrm{S}_{1}}^{45^{\circ}}-I_{\text {out, } \mathrm{S}_{1}}^{90^{\circ}}-I_{\text {out, } \mathrm{S}_{1}}^{0^{\circ}}}\right)
$$

By carrying out the derivation in a similar manner, the phase retardation caused by the presence of the pure BK7 substrate in the $\mathrm{S}_{2}$ track can be expressed as

$$
\Delta_{\mathrm{S}_{2}}=\tan ^{-1}\left(\frac{I_{\text {out, } \mathrm{S}_{2}}^{0^{\circ}}-I_{\text {out, } \mathrm{S}_{2}}^{90^{\circ}}}{2 I_{\text {out, } \mathrm{S}_{2}}^{45^{\circ}}-I_{\text {out, } \mathrm{S}_{2}}^{90^{\circ}}-I_{\text {out, } \mathrm{S}_{2}}^{0^{\circ}}}\right)
$$

Finally, we can find the phase difference between those two tracks by subtracting Eq. (17) from Eq. (16) as 


$$
\Delta_{\mathrm{S}}=\tan ^{-1}\left(\frac{I_{\text {out, } \mathrm{S}_{1}}^{0^{\circ}}-I_{\text {out, } \mathrm{S}_{1}}^{90^{\circ}}}{2 I_{\text {out, } \mathrm{S}_{1}}^{45^{\circ}}-I_{\text {out, } \mathrm{S}_{1}}^{90^{\circ}}-I_{\text {out, } \mathrm{S}_{1}}^{0^{\circ}}}\right)-\tan ^{-1}\left(\frac{I_{\text {out, } \mathrm{S}_{2}}^{0^{\circ}}-I_{\text {out, } \mathrm{S}_{2}}^{90^{\circ}}}{2 I_{\text {out, } \mathrm{S}_{2}}^{45^{\circ}}-I_{\text {out, } \mathrm{S}_{2}}^{90^{\circ}}-I_{\text {out, } \mathrm{S}_{2}}^{0^{\circ}}}\right)
$$

Note that the simultaneous signal from the reference track (the beam passing a pure BK7 substrate) provides both initial orientation and phase, which are essentially used to identify intensities at specific orientations and work out the phase shift introduced by the thin-film sample.

\subsection{Film thickness determination}

The transmitted light $T$ under various conditions can be carefully examined [22]. The phase retardation $\Delta_{\mathrm{S}}$, as a function of the film thickness, can be given by $4 \pi n_{1} d_{1} / \lambda$, where $n_{1}$ is the refractive index of the deposited film $\left(2.10\right.$ for $\left.\mathrm{WO}_{3}\right)$ [23],$d_{1}$ represents the film thickness, and $\lambda$ denotes the light wavelength $(632.8 \mathrm{~nm})$. Because the phase retardation $\Delta_{\mathrm{S}}$ obtained from Eq. (18) is in the form of a tangent function that repeats periodically every $180^{\circ}$ or $\pi$ radians, the phase shift satisfying the condition can be written in terms of the order number $m$ as $\Delta_{m}=\Delta_{\mathrm{S}}+m \pi$. Further, the phase shift $\Delta_{\mathrm{S}}$ in Eq. (18) can be accumulated or increased to match the measured $\Delta_{\mathrm{S}}$ by adding integer multiples of $\pi$, represented as $m \pi$. Here, $m$ denotes the cumulative integer chosen as the sign changes from negative to positive, i.e., it specifies the number of periods of the tangent function of $\Delta_{\mathrm{S}}$. Prior to calculating the thickness $d_{1}$, this relation must be satisfied to determine the exact order of the periodic tangent function of $\Delta_{\mathrm{S}}$ [24]. The parameter $d_{\text {cutoff }}$ is employed to determine the appropriate value of the order number $m$ when the deposition time $t_{\mathrm{d}}$ and approximate deposition rate $R_{\mathrm{d}}$ are known. In this study, $R_{\mathrm{d}}$ from the commercial UHV sputtering system used (AJA International Inc., ATC $2000-\mathrm{F}$ ) is $30 \mathrm{~nm} / \mathrm{min}$. The cutoff thickness $d_{\text {cutoff }}$, at the point at which the second cycle begins $\left(\Delta_{\mathrm{S}}=\pi\right)$, can be expressed as $\lambda / 4 n_{1}$. Accordingly, the appropriate value of $m$ can be determined using $t_{\mathrm{d}} R_{\mathrm{d}} / d_{\text {cutoff }}$. For $\mathrm{WO}_{3}$ with a refractive index of 2.10 and a red laser having wavelength of $632.8 \mathrm{~nm}, d_{\text {cutoff }}$ is found to be $70 \mathrm{~nm}$. According to the information, the values of $m$ at 10,15 , and $20 \mathrm{~min}$ with rate $R_{\mathrm{d}}=30 \mathrm{~nm} / \mathrm{min}$ are 4 , 6 , and 8, respectively. Eventually, the thin-film thickness $d_{1}$ can be determined with an appropriate value of $m$ as [19]

$$
d_{1}=\frac{\Delta_{m} \lambda}{4 \pi n_{1}}
$$

\section{Signal treatment algorithm to evaluate phase retardation}

Signal processing was employed to determine both the phase shifts given by Eqs. (16) and (17) between the parallel-polarized light beams in the two tracks. A digital oscilloscope (Yokogawa DLM2000) was used to obtain the desired information. The optimized amplitude and frequency of a modulating sinusoidal trigger signal were set at 
$1.6 \mathrm{~V}$ and $41 \mathrm{~Hz}$, respectively. The trigger function from the oscilloscope was carefully adjusted to stabilize signals from reference and sample tracks to be viewed clearly on the screen. We then produced the unbalanced phase by inserting a general WP inside the twin paths. Figure 2 shows both the signal intensities acquired when $\mathrm{WO}_{3}$ thin-film samples on BK7 substrate and bare BK7 substrate are inserted. At the output of the CI, the amplitude and phase information from the two tracks are related to the polarizer, which is oriented at $45^{\circ}$ with respect to the PB axis. Upon inserting thin-film samples
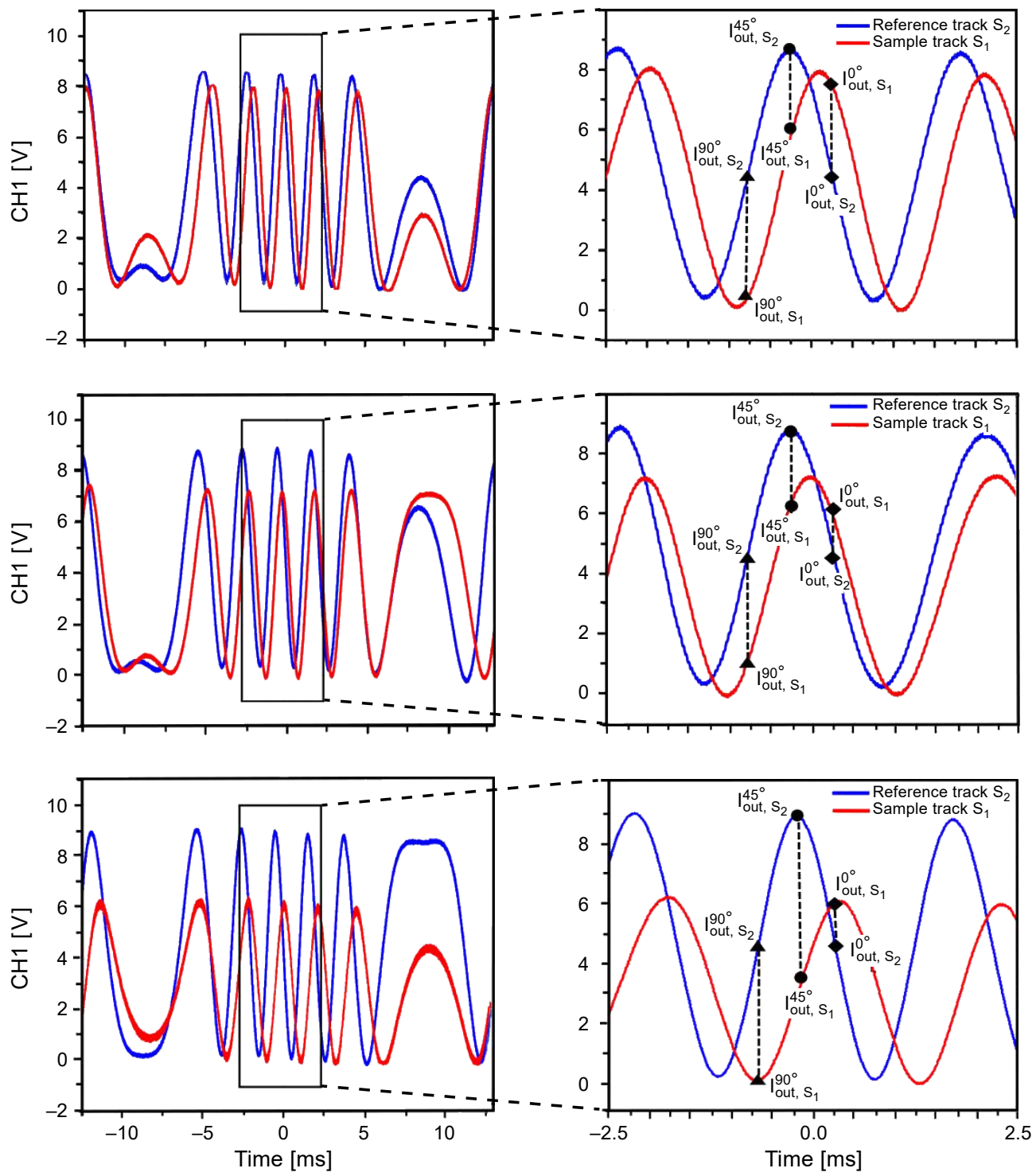

Fig. 2. Comparative differential relative phases of the output intensities of reference and sample waveforms for various deposition times: $10 \mathrm{~min}$ (a), $15 \mathrm{~min}$ (b), and $20 \mathrm{~min}$ (c). 
with deposition times of 10,15 , and 20 min into two tracks between $\mathrm{M}_{1}$ and $\operatorname{PZT}\left(\mathrm{M}_{2}\right)$, the output intensities of the parallel-polarized light are directly perturbed in terms of both amplitude and phase. This perturbation gives rise to the differential amplitudes and phase intensities between the two output signals. The sample and reference output waveforms are analyzed and the positions corresponding to three intensity points are checked. The orientation of the dual RPL is automatically rotated by $\theta$ due to the PZT modulation. The specific intensities of the two tracks can then be clearly observed when the polarizer is set at $45^{\circ}$ with respect to the $\mathrm{PB}$ axis. The outputs of the light intensities of the two sets are denoted by sample waveforms $I_{\text {out, }}^{0^{\circ}}, I_{\text {out, }}^{45_{1}^{\circ}}$, and $I_{\text {out, }}^{90^{\circ}} \mathrm{S}_{1}$, and reference waveforms $I_{\text {out, }}^{0^{\circ}}, I_{\text {out, }}^{45_{2}^{\circ}}$, and $I_{\text {out, }}^{90^{\circ}} \mathrm{S}_{2}$. Figures $2 \mathbf{a}-2 \mathbf{c}$ depict waveforms obtained after inserting a thin-film sample. Equation (18) is employed to evaluate the differential phase retardations $\Delta_{\mathrm{S}}$ that can be converted into the thin-film thicknesses using Eq. (19).

\section{Experimental results and discussion}

The degree of polarization (DOP) values of the dual RPL from the two tracks of the CI were determined to ensure the quality of the generated rotating polarized beams, which is very crucial for thin-film thickness measurements. A signal algorithm program was utilized to obtain the differential phase retardation (later converted to the film thicknesses). The film thickness read-outs obtained from the CI setup were compared with the ones acquired using conventional techniques such as variable-angle spectroscopic ellipsometry (VASE; J.A. Woollam) and field-emission scanning electron microscopy (FE-SEM; Hitachi S-4700) measurements.

\subsection{Film thickness determination}

The expected twin output light beams from both tracks of the CI are identically linearly polarized beams. Ideally, the perfectly polarized light beams obtained from both outputs have a degree of polarization (DOP) of $100 \%$. The characteristics of the dual beams were evaluated in terms of the four Stokes parameters. The output beam intensities yielded four terms of the Stokes parameters for the sample track as $S_{0}=11.237 \pm 0.080 \mathrm{~V}$, $S_{1}=11.210 \pm 0.080 \mathrm{~V}, S_{2}=-0.028 \pm 0.114 \mathrm{~V}$, and $S_{3}=0.009 \pm 0.035 \mathrm{~V}$. The corresponding values for the reference track were $S_{0}=11.134 \pm 0.066 \mathrm{~V}, S_{1}=11.116 \pm 0.064 \mathrm{~V}$, $S_{2}=-0.039 \pm 0.069 \mathrm{~V}$, and $S_{3}=0.015 \pm 0.015 \mathrm{~V}$. These parameters were processed to obtain the DOP [18] of $\mathrm{D}_{1}$ and $\mathrm{D}_{2}$ for the generated dual RPL as $99.72 \%$ and $99.83 \%$ for sample and reference tracks, respectively. These results clearly indicate that the states of the output dual beams from the CI system are extremely close to those of the ideal linearly polarized light beams. Subsequently, $\mathrm{WO}_{3}$ thin-film samples of different thicknesses were characterized using the CI setup. The samples of interest were inserted in the light-beam path of the sample track between $\operatorname{PZT}\left(\mathrm{M}_{2}\right)$ and $\mathrm{M}_{1}$. Both the output light intensities detected by $\mathrm{D}_{1}$ and $\mathrm{D}_{2}$ in terms of the voltages were processed in the algorithm described in Section 3, so that the phase shifts $\Delta_{\mathrm{S} 1}$ and $\Delta_{\mathrm{S} 2}$ needed could be achieved. The three output light intensities listed in the Table are the average values 
$\mathrm{T}$ a b 1 e. Average maximum intensities under various study conditions.

\begin{tabular}{lllllll}
\hline \multirow{2}{*}{ Sample } & \multicolumn{6}{l}{ Average maximum intensity [V] } \\
\cline { 2 - 7 } & $I_{\text {out, } \mathrm{S}_{1}}^{0^{\circ}}$ & $I_{\text {out, } \mathrm{S}_{2}}^{0^{\circ}}$ & $I_{\text {out, } \mathrm{S}_{1}}^{45^{\circ}}$ & $I_{\text {out, } \mathrm{S}_{2}}^{45^{\circ}}$ & $I_{\text {out, } \mathrm{S}_{1}}^{90^{\circ}}$ & $I_{\text {out, } \mathrm{S}_{2}}^{90^{\circ}}$ \\
\hline $\mathrm{WO}_{3}=10 \mathrm{~min}$ & 7.63 & 4.53 & 6.18 & 8.75 & 0.52 & 4.40 \\
$\mathrm{WO}_{3}=15 \mathrm{~min}$ & 6.38 & 4.75 & 6.25 & 8.84 & 0.94 & 4.62 \\
$\mathrm{WO}_{3}=20 \mathrm{~min}$ & 6.01 & 4.66 & 3.75 & 8.94 & 0.12 & 4.58 \\
\hline
\end{tabular}

of the measured maximum intensities depicted in Figs. $2 \mathbf{a}-2 \mathbf{c}$ for various linear polarizer orientations and deposition times.

The phase retardations introduced by the inserted samples for deposition times of 10,15 , and $20 \mathrm{~min}$ were found to be $1.08,0.79$, and $1.33 \mathrm{rad}$, respectively. To determine the appropriate $m$ values, the maximal and minimal $T$ values were tracked along with the phase retardation. The thin-film thickness can be calculated using Eq. (19), e.g., $d_{1}(10 \mathrm{~min})=(1.08+4 \pi) \lambda / 4 \pi n_{1}=327.2 \mathrm{~nm}, d_{2}(15 \mathrm{~min})=(0.79+6 \pi) \lambda / 4 \pi n_{1}=$ $=470.9 \mathrm{~nm}$, and $d_{3}(20 \mathrm{~min})=(1.33+8 \pi) \lambda / 4 \pi n_{1}=634.5 \mathrm{~nm}$. The measured thin-film thicknesses using the CI system for deposition times of 10, 15, and 20 min were found to be $327.2,470.9$, and $634.5 \mathrm{~nm}$, respectively.

\subsection{Spectroscopic ellipsometry (SE) and FE-SEM analysis}

The values obtained from the VASE for the $\mathrm{WO}_{3}$ thin-film and the Tauc-Lorentz oscillator [25] were used to plot the fitting curves of the ellipsometric parameters for different deposition times. The VASE results showed that the $\mathrm{WO}_{3}$ thin-film-sample thicknesses were $298.9,440.3$, and $595.5 \mathrm{~nm}$ for deposition times of 10, 15, and $20 \mathrm{~min}$, respectively. The samples were then placed under the field-emission scanning electron microscopy (FE-SEM) system to verify the results.

Figure 3 shows the cross-sectional FE-SEM micrographs of the $\mathrm{WO}_{3}$ thin-films on the BK7 substrate for various deposition times. The films are compact, homogeneous, and perfectly adherent to the BK7 substrate. The average thicknesses obtained from the
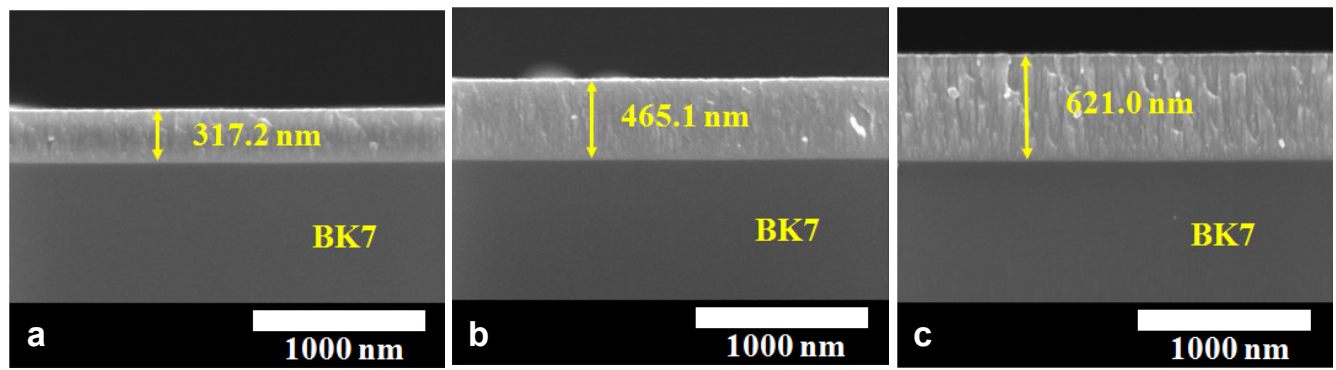

Fig. 3. Cross-sectional field-emission scanning electron microscopy (FE-SEM) images of $\mathrm{WO}_{3}$ thin-film -sample thicknesses for deposition times of $10 \mathrm{~min}(\mathbf{a}), 15 \mathrm{~min}(\mathbf{b})$, and $20 \mathrm{~min}$ (c). 
image analysis of the cross-section sample for deposition times of 10, 15, and 20 min were $313.6,465.3$, and $615.1 \mathrm{~nm}$, respectively.

\subsection{Result comparison and discussion}

The film thickness measurements achieved with CI, SE, and FE-SEM were compared. The thickness values obtained using the CI with SE and CI with FE-SEM exhibited reading differences of $7.31 \% / 3.15 \%, 6.95 \% / 1.25 \%$, and $6.54 \% / 2.17 \%$ with respect to the average readings from the standard tools for deposition times of 10, 15, and $20 \mathrm{~min}$, respectively. The longer the deposition time, the lower was the reading error. This could be explained by the deposition rate being unsteady at the beginning and becoming more linear with the passage of time. Nevertheless, the results showed no substantial deviation between the SE, FE-SEM, and CI methods.

\section{Conclusions}

This study reports a new scheme to determine the thin-film thickness of a transparent sample using a CI system. The dual RPL produced by our CI setup possessed an excellent degree of polarization. Samples with smooth $\mathrm{WO}_{3}$ thin-films were prepared by using magnetron sputtering. The films exhibited low absorption at high deposition rates over deposition times of 10,15 , and $20 \mathrm{~min}$. To verify the performance of the CI setup, the experimental results obtained with our CI system were compared with the ones obtained with standard VASE and FE-SEM techniques. There was no significant difference between the three sets of results. With our approach, it is possible to produce suitable dual RPL, accurately investigate thin-film thickness, and characterize the phase retardation of optical devices such as polarizers, wave retarders, and liquid-crystal variable wave retarders.

Acknowledgements - The authors would like to acknowledge the contribution of the Thai Government Science and Technology Scholarship for funding the project. The authors would also like to thank Dr. Mati Horprathum for his time and advice, providing film samples, and performing film measurements.

\section{References}

[1] Chakraborty S., Bhattacharya K., Low-level birefringence measurement by cyclic-path polarization interferometer, Applied Optics 55(21), 2016, pp. 5634-5639, DOI: 10.1364/AO.55.005634.

[2] Flores Muñoz V.H., Toto-Arellano N.I., López-Ortiz B., Martínez García A., Rodríguez-Zurita G., Measurement of red blood cell characteristic using parallel phase shifting interferometry, Optik 126(24), 2015, pp. 5307-5309, DOI: 10.1016/j.ijleo.2015.09.019.

[3] Kumar Y.P., CHATterJeE S., Thickness measurement of transparent glass plates using a lateral shearing cyclic path optical configuration setup and polarization phase shifting interferometry, Applied Optics 49(33), 2010, pp. 6552-6557, DOI: 10.1364/AO.49.006552.

[4] Rothau S., Kellermann C., Mayer S., Mantel K., Lindlein N., Polarization and phase-shifting interferometry for arbitrary, locally varying polarization states, Applied Optics 56(5), 2017, pp. 1422 -1430, DOI: 10.1364/AO.56.001422. 
[5] Malacara D., Phase shifting interferometry, Revista Mexicana de Física 36(1), 1990, pp. 6-22.

[6] Meneses-Fabian C, Rivera-Ortega U., Phase-shifting interferometry by wave amplitude modulation, Optics Letters 36(13), 2011, pp. 2417-2419, DOI: 10.1364/OL.36.002417.

[7] Indebetouw G., KLysubun P., Measurement of induced birefringence in film samples using a balanced polarization ring interferometer, Optics Communications 151(4-6), 1998, pp. 203-206, DOI: 10.1016/S0030-4018(98)00087-X.

[8] Bhaduri B., KRishna Mohan N., Kothiyal M.P., Cyclic-path digital speckle shear pattern interferometer: use of polarization phase-shifting method, Optical Engineering 45(10), 2006, article 105604, DOI: $10.1117 / 1.2361194$.

[9] Shagam R.N., Wyant J.C., Optical frequency shifter for heterodyne interferometers using multiple rotating polarization retarders, Applied Optics 17(19), 1978, pp. 3034-3035, DOI: 10.1364/AO.17. $\underline{003034}$.

[10] Kothiyal M.P., Delisle C., Shearing interferometer for phase shifting interferometry with polarization phase shifter, Applied Optics 24(24), 1985, pp. 4439-4442, DOI: 10.1364/AO.24.004439.

[11] Toto-Arellano N.-I., Serrano-García D.-I., Martínez-García A., Parallel two-step phase shifting interferometry using a double cyclic shear interferometer, Optics Express 21(26), 2013, pp. 31983 -31989, DOI: 10.1364/OE.21.031983.

[12] Zhang Z., Zhang Q., Cheng T., Gao J., Wu X., Wave-plate phase shifting method, Optical Engineering 52(10), 2013, article 103109, DOI: 10.1117/1.OE.52.10.103109.

[13] Suja Helen S., Kothiyal M.P., Sirohi R.S., Achromatic phase shifting by a rotating polarizer, Optics Communications 154(5-6), 1998, pp. 249-254, DOI: 10.1016/S0030-4018(98)00292-2.

[14] Lechuga L.G., Toto-Arellano N.I., Flores Muñoz V.H., Martínez-García A., Zurita G.R., Phase shifting interferometry using a coupled cyclic path interferometers, [In] Emerging Challenges for Experimental Mechanics in Energy and Environmental Applications, Proceedings of the 5th International Symposium on Experimental Mechanics and 9th Symposium on Optics in Industry (ISEM-SOI), 2015, Martínez-García A., Furlong C., Barrientos B., Pryputniewicz R. [Eds.],Conference Proceedings of the Society for Experimental Mechanics Series, Springer, Cham, 2017, pp. 65-69,

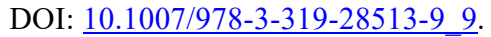

[15] Wang L., Liu L., Luan Z., Sun J., Zhou Y., Polarization phase-shifting Jamin shearing interferometer, Optik 121(4), 2010, pp. 358-361, DOI: 10.1016/j.ijleo.2008.07.023.

[16] Chakraborty S., Bhattacharya K., Real-time edge detection by cyclic-path polarization interferometer, Applied Optics 53(4), 2014, pp. 727-730, DOI: 10.1364/AO.53.000727.

[17] LiU X., GAO Y., CHANG M., A new lateral shearing interferometer for precision surface measurement, Optics and Lasers in Engineering 47(9), 2009, pp. 926-934, DOI: 10.1016/j.optlaseng.2009.03.019.

[18] Pawong C., Chitaree R., Soankwan C., The rotating linearly polarized light from a polarizing Mach-Zehnder interferometer: production and applications, Optics and Laser Technology 43(3), 2011, pp. 461-468, DOI: 10.1016/j.optlastec.2010.06.020.

[19] Kaewon R., Pawong C., Chitaree R., Bhatranand A., Polarization phase-shifting technique for the determination of a transparent thin film's thickness using a modified Sagnac interferometer, Current Optics and Photonics 2(5), 2018, pp. 474-481.

[20] TATAM R.P., Jones J.D.C., JACKSON D.A., Optical polarization state control schemes using fibre optics or Bragg cells, Journal of Physics E: Scientific Instruments 19(9), 1986, pp. 711-717, DOI: 10.1088/0022-3735/19/9/013.

[21] Sarkar S., Bhattacharya K., Polarization phase shifting cyclic interferometer for surface profilometry of non-birefringent phase samples, Journal of Modern Optics 60(3), 2013, pp. 185-189, DOI: $10.1080 / 09500340.2013 .765051$.

[22] Manifacier J.C., Gasiot J., Fillard J.P., A simple method for the determination of the optical constants $n, k$ and the thickness of a weakly absorbing thin film, Journal of Physics E: Scientific Instruments 9(11), 1976, pp. 1002-1004, DOI: 10.1088/0022-3735/9/11/032. 
[23] Sun X., Liu Z., Cao H., Effects of film density on electrochromic tungsten oxide thin films deposited by reactive dc-pulsed magnetron sputtering, Journal of Alloys and Compounds 504, 2010, pp. s418 -s421, DOI: $10.1016 /$ j.jallcom.2010.03.155.

[24] Zhao C., TAn J., TANG J., LiU T., Liu J., Confocal simultaneous phase-shifting interferometry, Applied Optics 50(5), 2011, pp. 655-661, DOI: 10.1364/AO.50.000655.

[25] Franke E., Trimble C.L., DeVries M.J., Woollam J.A., Schubert M., Frost F., Dielectric function of amorphous tantalum oxide from the far infrared to the deep ultraviolet spectral region measured by spectroscopic ellipsometry, Journal of Applied Physics 88(9), 2000, pp. 5166-5174, DOI: 10.1063/1.1313784.

Received December 17, 2018 